\title{
Modellierung eines Halbraums mit sphärischem oder zylinderförmigem Hohlraum für dreidimensionale Boden- Bauwerk-Interaktion
}

\author{
J. Freisinger M.Sc., Prof. Dr.-Ing. G. Müller
}

Technische Universität München, Lehrstuhl für Baumechanik

\section{Kurzfassung}

Dieser Beitrag präsentiert eine Berechnungsmethode für die Wellenausbreitung infolge einer dynamischen Belastung in einem elastischen Halbraum mit zylinderförmigem oder sphärischem Hohlraum, Graben oder kugelförmigem Einschnitt. Durch die Superposition der Grundlösungen der Integraltransformationsmethode (Halbraum, Vollraum mit zylinderförmigem Hohlraum und Vollraum mit sphärischem Hohlraum) erhält man für diese Systeme eine semianalytische Lösung im Wellenzahl-Frequenzraum. Daraus resultieren schließlich die wellenzahlabhängigen Nachgiebigkeiten an der Oberfläche des Halbraums sowie des zylindrischen oder sphärischen Einschlusses. Diese Nachgiebigkeiten können dann mit der Finiten-Elemente-Methode gekoppelt werden, so dass beliebige komplexe Strukturen in das Bodenmodell eingebettet werden können.

\section{Einleitung}

Für eine zuverlässige Vorhersage und Beurteilung von Erschütterungen und Vibrationen, infolge dynamischer Einwirkungen, muss zur Erfassung der komplexen Interaktion zwischen Boden und Bauwerk das gesamte Emissions-Transmissions-Immissionssystem modelliert werden. Eine geeignete Methode zur Beschreibung des Bodens als Transmissionsmedium mit infiniter Ausdehnung stellt die Integral-Transformations-Methode (ITM) dar, da diese geschlossenen Lösungen für verschiedene Fundamentalsysteme liefert und damit künstliche Reflektionen an Bereichsrändern vermieden werden können. Allerdings können nur einfache Geometrien mit Hilfe der Fundamentallösungen beschrieben werden, weshalb die Modellierung einer komplexen Gebäudestruktur als Immissionsmedium oder einer komplexen Lasteinwirkungsumgebung als Emissionsmedium mit der ITM nicht möglich ist. Solche Strukturen können demgegenüber mit der Finite-Elemente-Methode (FEM) abgebildet werden, die mit Wellenreflektionen an den Gebietsrändern in Verbindung steht und so den unendlich ausgedehnten Boden nur mit Einschränkungen abbilden kann. Eine Verknüpfung beider Methoden ermöglicht es, die Vorteile von beiden Verfahren zu kombinieren und stellt daher eine geeignete Möglichkeit zur Modellierung der Boden-Bauwerk-Interaktion (BBI) dar. 
Zunächst werden dazu die Lösungen der Integraltransformationsmethode für den Halbraum mit zylinderförmigem Hohlraum und Halbraum mit sphärischem Hohlraum eingeführt. Da geschlossene Lösungen nur für die Fundamentalsysteme Halbraum, Vollraum mit zylinderförmigem Hohlraum und Vollraum mit sphärischem Hohlraum vorliegen [1], wird die Lösung aus einer Superposition dieser Grundlösungen konstruiert. Im Anschluss werden die wellenzahlabhängigen Nachgiebigkeiten an den Oberflächen der Hohlräume mit der FinitenElemente-Methode gekoppelt, mit welcher beliebig komplexe Strukturen und ein Teil des daran anschließenden Bodens modelliert werden können [2].

\section{Grundlösungen in der Elastodynamik}

\subsection{Grundlösung für den Halbraum}

Das dynamische Gleichgewicht in einem dreidimensionalen, linear elastischen, homogenen und isotropen Kontinuum wird durch die Lamé Gleichung beschrieben, welche ein System von drei partiellen, gekoppelten Differentialgleichungen darstellt.

$$
\left.\mu u^{i}\right|_{j} ^{j}+\left.(\lambda+\mu) u^{j}\right|_{j} ^{i}-\rho \ddot{u}^{i}=0
$$

Mit dem Verschiebungsfeld $u^{i}$, den Lamé Konstanten $\lambda, \mu$ für das Material sowie der Dichte $\rho$. Die Lamé Gleichung kann mit Hilfe des Satzes von Helmholtz entkoppelt werden. Dazu wird das Verschiebungsfeld $u^{i}$ in ein Skalarpotential $\Phi$ und ein Vektorpotential $\boldsymbol{\Psi}$ zerlegt:

$$
u^{i}=\left.\phi\right|^{i}+\left.\Psi_{l}\right|_{k} \varepsilon^{i k l}
$$

Die resultierenden partiellen Differentialgleichungen können mit Hilfe einer dreifachen Fouriertransformation vom Orts- $(x, y)$ in den Wellenzahlraum $\left(k_{x}, k_{y}\right)$ und vom Zeitbereich $t$ in den Frequenzraum $\omega$ in ein System gewöhnlicher Differentialgleichungen überführt werden:

$$
\begin{aligned}
& {\left[-k_{x}{ }^{2}-k_{y}{ }^{2}+k_{p}{ }^{2}+\frac{\partial^{2}}{\partial z^{2}}\right] \widehat{\Phi}\left(k_{x}, k_{y}, z, \omega\right)=0} \\
& {\left[-k_{x}{ }^{2}-k_{y}{ }^{2}+k_{s}{ }^{2}+\frac{\partial^{2}}{\partial z^{2}}\right] \widehat{\Psi}_{i}\left(k_{x}, k_{y}, z, \omega\right)=0}
\end{aligned}
$$

mit den Wellenzahlen $k_{p}=\omega / c_{p}$ und $k_{s}=\omega / c_{s}$ der Kompressions- und der Scherwelle. Dieses kann unter Verwendung eines Exponentialansatzes für das Skalar- und die Vektorpotentiale gelöst werden.

mit

$$
\begin{aligned}
& \widehat{\Phi}=A_{1} e^{\lambda_{1} z}+A_{2} e^{-\lambda_{1} z} \quad \text { und } \quad \widehat{\Psi}_{i}=B_{i 1} e^{\lambda_{2} z}+B_{i 2} e^{-\lambda_{2} z} \\
& \lambda_{1}=\sqrt{k_{x}^{2}+k_{y}^{2}-k_{p}^{2}} \quad \text { und } \quad \lambda_{2}=\sqrt{k_{x}^{2}+k_{y}^{2}-k_{s}^{2}}
\end{aligned}
$$

Die unbekannten Koeffizienten $A_{1}, A_{2}, B_{i 1}, B_{i 2}$ können mit Hilfe der Randbedingungen an der Halbraumoberfläche und der Sommerfeldschen Abstrahlbedingung bestimmt werden [4]. 


\subsection{Grundlösung für den Vollraum mit zylinderförmigem Hohlraum}

Für die Lösung des Systems Vollraum mit zylindrischem Hohlraum wird die Lamé Differentialgleichung in Zylinderkoordinaten transformiert.

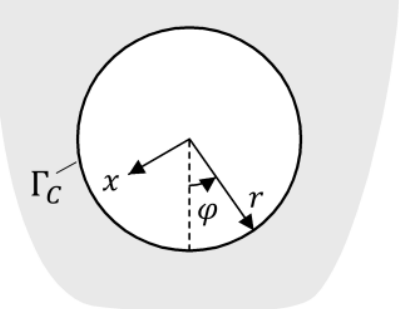

Abbildung 1: Vollraum mit zylindrischem Hohlraum und Zylinderkoordinatensystem.

Nach der Entkopplung mit dem Satz von Helmholtz wird das Vektorpotential $\boldsymbol{\Psi}$ durch zwei unabhängige skalare Funktionen $\psi$ und $\chi$ ausgedrückt [3].

$$
\boldsymbol{\Psi}=\psi \boldsymbol{g}^{\mathbf{1}}+\left.\chi\right|^{j} \epsilon_{i j 1} \boldsymbol{g}^{i}
$$

Um wiederum drei gewöhnliche Differentialgleichungen zu erhalten, wird eine zweifache Fouriertransformation in den Wellenzahlenfrequenzraum $\left(x \circ-k_{x}, t \circ-\omega\right)$ und eine Fourierreihenentwicklung in Umfangsrichtung $\varphi$ durchgeführt.

$$
\begin{aligned}
& {\left[-k_{x}^{2}+\frac{\partial^{2}}{\partial r^{2}}+\frac{1}{r} \frac{\partial}{\partial r}-\frac{n^{2}}{r^{2}}+k_{p}^{2}\right] \widehat{\Phi}\left(k_{x}, r, n, \omega\right)=0} \\
& {\left[-k_{x}^{2}+\frac{\partial^{2}}{\partial r^{2}}+\frac{1}{r} \frac{\partial}{\partial r}-\frac{n^{2}}{r^{2}}+k_{s}^{2}\right] \hat{\psi}\left(k_{x}, r, n, \omega\right)=0} \\
& {\left[-k_{x}^{2}+\frac{\partial^{2}}{\partial r^{2}}+\frac{1}{r} \frac{\partial}{\partial r}-\frac{n^{2}}{r^{2}}+k_{s}^{2}\right] \hat{\chi}\left(k_{x}, r, n, \omega\right)=0}
\end{aligned}
$$

mit

$$
k_{\alpha}=\sqrt{k_{p}^{2}-k_{x}^{2}} \quad \text { and } \quad k_{\beta}=\sqrt{k_{s}^{2}-k_{x}^{2}}
$$

Die Lösung für $\widehat{\Phi}, \widehat{\Psi}$ and $\widehat{X}$ kann mit Hilfe von Hankel Funktionen beschrieben werden [3]:

$$
\begin{aligned}
& \widehat{\Phi}=C_{1 n} H_{n}^{(1)}\left(k_{\alpha} r\right)+C_{4 n} H_{n}^{(2)}\left(k_{\alpha} r\right) \\
& \widehat{\Psi}=C_{2 n} H_{n}^{(1)}\left(k_{\beta} r\right)+C_{5 n} H_{n}^{(2)}\left(k_{\beta} r\right) \\
& \widehat{X}=C_{3 n} H_{n}^{(1)}\left(k_{\beta} r\right)+C_{6 n} H_{n}^{(2)}\left(k_{\beta} r\right)
\end{aligned}
$$

Die Bestimmung der Unbekannten erfolgt wiederum mit Hilfe der lokalen Randbedingungen am Hohlraumrand sowie der Sommerfeldschen Abstrahlbedingung.

\subsection{Grundlösung für den Vollraum mit sphärischem Hohlraum}

Die Lösung für den Vollraum mit sphärischem Hohlraum erfolgt in Kugelkoordinaten $(r, \vartheta, \varphi)$. 

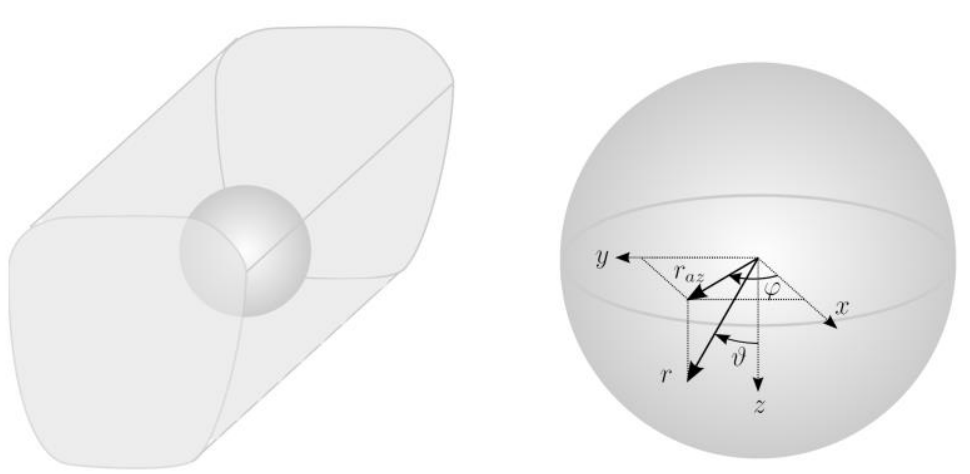

Abbildung 2: Vollraum mit sphärischem Hohlraum (links) und Kugelkoordinaten (rechts).

Analog zum zylindrischen Koordinatensystem wird das Vektorpotential $\boldsymbol{\Psi}$ durch zwei unabhängige skalare Funktionen $\psi$ und $\chi$ ausgedrückt:

$$
\boldsymbol{\Psi}=r \psi \boldsymbol{g}^{\mathbf{1}}+\left.(r \chi)\right|^{j} \epsilon_{i j 1} \boldsymbol{g}^{\boldsymbol{i}}
$$

Im Folgenden werden das Skalarpotential $\Phi$ und die skalaren Funktionen $\psi$ und $\chi$ in eine Reihe von Kugelflächenfunktionen zerlegt:

$$
\begin{aligned}
& \Phi(r, \vartheta, \varphi, t)=\sum_{m=0}^{\infty} \sum_{l=-m}^{m} \Phi(r, m, l, t) Y_{m}^{l}(\vartheta, \varphi) \\
& \psi(r, \vartheta, \varphi, t)=\sum_{m=0}^{\infty} \sum_{l=-m}^{m} \psi(r, m, l, t) Y_{m}^{l}(\vartheta, \varphi) \\
& \chi(r, \vartheta, \varphi, t)=\sum_{m=0}^{\infty} \sum_{l=-m}^{m} \chi(r, m, l, t) Y_{m}^{l}(\vartheta, \varphi)
\end{aligned}
$$

Die Kugelflächenfunktionen sind definiert als:

$$
Y_{m}^{l}(\vartheta, \varphi)=\sqrt{\frac{2 m+1}{2} \frac{(m-l) !}{(m+l) !}} P_{m}^{l}(\cos (\vartheta)) e^{i l \varphi}
$$

Das Einsetzen der Reihenentwicklung in die Wellengleichungen in Kugelkoordinaten, ein Koeffizientenvergleich und eine anschließende Fouriertransformation in den Frequenzbereich $(t \circ \cdots \omega)$ führt zu den folgenden drei entkoppelten, gewöhnlichen Differentialgleichungen [2]:

$$
\begin{aligned}
& {\left[\frac{\partial^{2}}{\partial r^{2}}+\frac{2}{r} \frac{\partial}{\partial r}+\left(k_{p}{ }^{2}-\frac{m(m+1)}{r^{2}}\right)\right] \widehat{\Phi}(r, m, l, \omega)=0} \\
& {\left[\frac{\partial^{2}}{\partial r^{2}}+\frac{2}{r} \frac{\partial}{\partial r}+\left(k_{s}{ }^{2}-\frac{m(m+1)}{r^{2}}\right)\right] \widehat{\Psi}(r, m, l, \omega)=0} \\
& {\left[\frac{\partial^{2}}{\partial r^{2}}+\frac{2}{r} \frac{\partial}{\partial r}+\left(k_{s}{ }^{2}-\frac{m(m+1)}{r^{2}}\right)\right] \widehat{X}(r, m, l, \omega)=0}
\end{aligned}
$$

Dies sind Differentialgleichungen vom Besselschen Typ und können mit Hilfe von sphärischen Hankel Funktionen erster und zweiter Art gelöst werden: 


$$
\begin{gathered}
\widehat{\Phi}(r, m, l, \omega)=C_{1 l m} h_{m}^{(1)}\left(\left|k_{p}\right| r\right)+C_{4 l m} h_{m}^{(2)}\left(\left|k_{p}\right| r\right) \\
\hat{\psi}(r, m, l, \omega)=C_{2 l m} h_{m}^{(1)}\left(\left|k_{s}\right| r\right)+C_{5 l m} h_{m}^{(2)}\left(\left|k_{s}\right| r\right) \\
\hat{\chi}(r, m, l, \omega)=C_{3 l m} h_{m}^{(1)}\left(\left|k_{s}\right| r\right)+C_{6 l m} h_{m}^{(2)}\left(\left|k_{s}\right| r\right)
\end{gathered}
$$

Die Bestimmung der Unbekannten erfolgt wiederum mit Hilfe der lokalen Randbedingungen am Hohlraumrand sowie der Sommerfeldschen Abstrahlbedingung.

\section{Superposition von Grundlösungen}

Im Folgenden wird die Lösung für den Halbraum mit sphärischem Hohlraum oder kugelförmigem Einschnitt präsentiert, die Lösung für den Halbraum mit zylindrischem Hohlraum oder Graben kann auf ähnliche Weise hergeleitet werden [2].

\subsection{Lösung für den Halbraum mit sphärischem HohIraum oder Einschnitt}

Für die Lösung des Systems eines Halbraums mit kugelförmigen Hohlraum oder eines Halbraums mit einer kugelförmigen Grube an der Halbraumoberfläche wird das Fundamentalsystem des Halbraums mit dem Fundamentalsystem des Vollraums mit sphärischem Hohlraum überlagert. Durch eine Fouriertransformation vom Zeit- in den Frequenzbereich $(t \circ \bullet \omega)$ kann das Problem quasistatisch gelöst werden. Alle Größen an der Halbraumoberfläche $(z=0)$ werden in Fourierreihen $\left(x \rightarrow o \cdot \Delta k_{x} y \rightarrow s \cdot \Delta k_{y}\right)$ und alle Größen am sphärischen Hohlraum $\left(r=R_{0}\right)$ in eine Reihe von Kugelflächenfunktionen zerlegt $(\vartheta, \varphi \rightarrow l, m)$. Unter Verwendung der Grundlösungen aus Abschnitt 2 können die Spannungen in kartesischen Koordinaten entlang der fiktiven sphärischen Hohlraumoberfläche $\delta \Gamma_{\mathrm{S}}$ infolge von dynamischen Lasten an der Oberfläche des Halbraums $\Lambda$ berechnet werden. Diese Spannungen werden dann in Kugelkoordinaten transformiert und in eine Reihe von Kugelflächenfunktionen zerlegt.

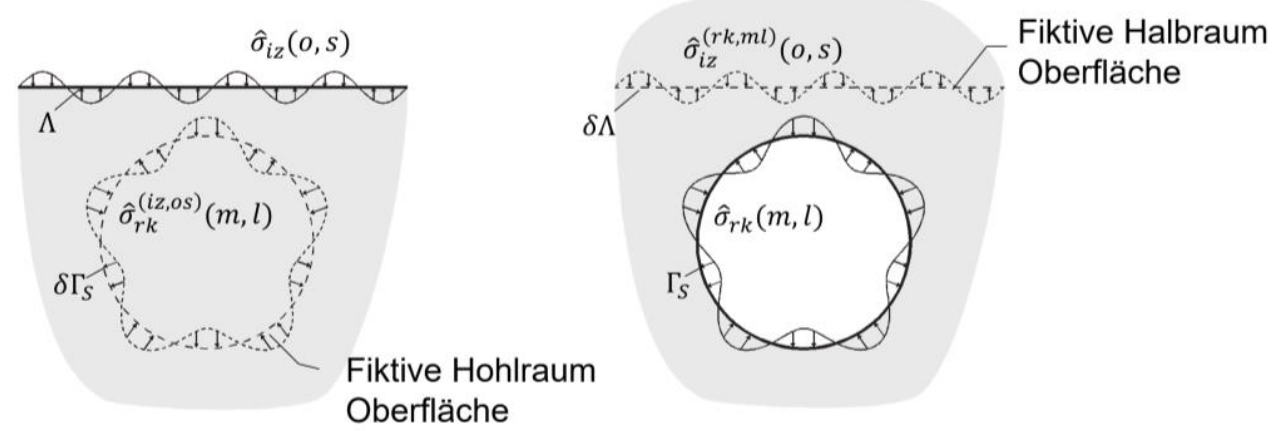

Abbildung 3: Substeme: Halbraum (links) und Vollraum mit sphärischem Hohlraum (rechts)

Damit ist es möglich für jeden kartesischen Einheitsspannungszustand an der Oberfläche $\hat{\sigma}_{i z}(o, s)$ die resultierenden Spannungen $\hat{\sigma}_{r k}^{(i z, o s)}(m, l)$ am sphärischen Hohlraumrand zu 
berechnen (mit $i=x, y, z$ und $k=R, \vartheta, \varphi$ ). Analog ist es möglich die Spannungen entlang der fiktiven Halbraumoberfläche $\delta \Lambda$ infolge einer dynamischen Last an der Hohlraumoberfläche $\Gamma_{\mathrm{S}}$ zu bestimmen. Transformiert man diese Spannungen in kartesische Koordinaten und entwickelt sie in Fourierreihen, bezüglich der $x-$ und $y-$ Koordinaten, erhält man die Spannungen an der fiktiven Halbraumoberfläche $\hat{\sigma}_{i z}^{(r k, m l)}(o, s)$ bedingt durch jeden sphärischen Einheitsspannungszustand $\hat{\sigma}_{r k}(m, l)$, welcher am sphärischen Hohlraum wirkt. Die Superposition der Spannungen an beiden Oberflächen muss die äußeren Randbedingungen erfüllen. Dazu müssen die Amplituden der Einheitsspannungszustände $C_{i z}(o, s)$ an $\Lambda$ und $C_{r k}(m, l)$ an $\Gamma_{\mathrm{S}}$ so bestimmt werden, dass die Summe den dort aufgebrachten äußeren Lasten $\hat{p}_{i, \Lambda}(s)$ auf $\Lambda$ und $\hat{p}_{k, \Gamma_{\mathrm{S}}}(n)$ auf $\Gamma_{\mathrm{S}}$ entspricht.

$$
\begin{aligned}
& C_{i z}(o, s) \hat{\sigma}_{i z}(o, s)+\sum_{M} \sum_{L} \sum_{K} C_{r k}(m, l) \hat{\sigma}_{i z}^{(r k, m l)}(o, s)=-\hat{p}_{i, \Lambda}(o, s) \\
& \sum_{O} \sum_{S} \sum_{I} C_{i z}(o, s) \hat{\sigma}_{r k}^{(i z, o s)}(m, l)+C_{r k}(m, l) \hat{\sigma}_{r k}(m, l)=-\hat{p}_{k, \Gamma}(m, l)
\end{aligned}
$$

Somit können alle Unbekannten $C_{i z}(o, s)$ und $C_{r k}(m, l)$ infolge einer beliebigen äußeren Last berechnet werden. Das Gleichungssystem in Matrixschreibweise ausgedrückt:

$$
\widehat{\boldsymbol{S}}_{\text {ITM }} \boldsymbol{C}=-\widehat{\boldsymbol{P}}_{\text {ITM }} \rightarrow C=-\widehat{\boldsymbol{S}}_{\text {ITM }}^{-1} \widehat{\boldsymbol{P}}_{\text {ITM }}
$$

Um die resultierenden Verschiebungen $\hat{u}$ an den Oberflächen zu berechnen müssen alle Verschiebungen resultierend aus den Einheitsspannungszuständen mit den bereits berechneten Unbekannten $\boldsymbol{C}$ skaliert und dann aufsummiert werden:

$$
\widehat{\boldsymbol{u}}_{\text {ITM }}=\widehat{V}_{\text {ITM }} C=-\widehat{V}_{\text {ITM }} \widehat{\boldsymbol{S}}_{\text {ITM }}^{-1} \widehat{\boldsymbol{P}}_{\text {ITM }}=\widehat{N}_{\text {ITM }} \widehat{\boldsymbol{P}}_{\text {ITM }}
$$

Damit ergibt die dynamische Flexibilitätsmatrix bzw. die dynamische Steifigkeitsmatrix zu:

$$
\widehat{N}_{I T M}=-\widehat{V}_{I T M} \widehat{S}_{I T M}^{-1} \quad \text { bzw. } \quad \widehat{K}_{\text {ITM }}=\widehat{N}_{I T M}^{-1}
$$

Das System Halbraum mit kugelförmigem Einschnitt kann auf ähnliche Weise gelöst werden. Dafür werden die Spannungen an der fiktiven Kugeloberfläche unterhalb der Halbraumoberfläche $\left(\delta \Gamma_{2 a}\right)$ infolge der Einheitsspannungszustände $C_{i z}(o, s)$ auf $\Lambda_{1}$ berechnet.

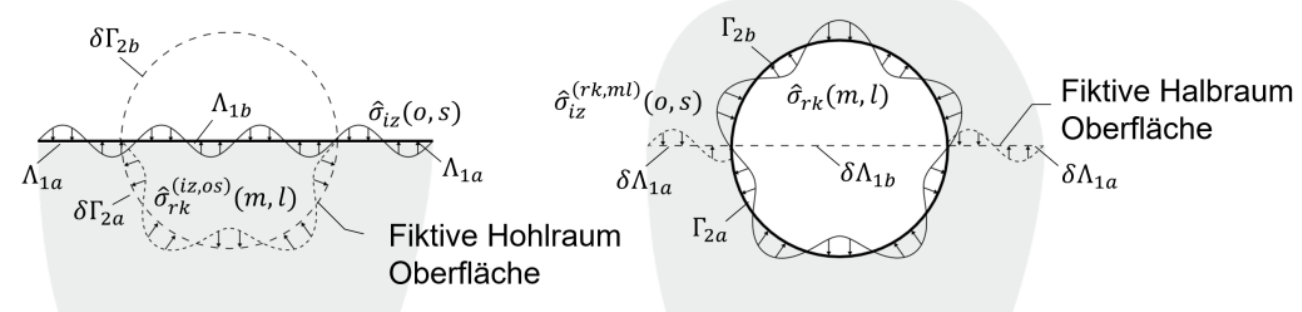

Abbildung 4: Subsysteme für Graben: Halbraum (links) und Vollraum mit sphärischem Hohlraum (rechts) 
Ebenso werden für die Spannungszustände $\hat{\sigma}_{r k}(m, l)$, welche auf der sphärischen Hohlraumoberfläche $\left(\Gamma_{2}\right)$ wirken, die Spannungen auf der fiktiven Halbraumoberfläche berechnet, welche außerhalb des Hohlraums liegen $\delta \Lambda_{1 a}$. Die Spannungen außerhalb bzw. innerhalb des jeweils betrachteten Bereichs $\left(\delta \Gamma_{2 b}\right.$ bzw. $\left.\delta \Lambda_{1 b}\right)$ müssen nicht berechnet werden, da die Randbedingungen nur an Rändern $\Lambda_{1 a}$ und $\Gamma_{2 a}$ eingehalten werden müssen. Da die Kopplung im $\left(k_{x}, k_{y}, z, \omega\right)$ bzw. $(R, m, l, \omega)$ Raum stattfindet, werden, um numerische Fehler bei den Reihenentwicklungen zu vermeiden, die Spannungen an den Rändern $\delta \Gamma_{2 b}$ und $\delta \Lambda_{1 b}$ mit Hilfe von Splines ergänzt, so dass keine Sprünge am Übergang von $\delta \Gamma_{2 a}$ zu $\delta \Gamma_{2 b}$ bzw. $\delta \Lambda_{1 a}$ zu $\delta \Lambda_{1 b}$ auftreten. Die Unbekannten können dann unter Verwendung der oben beschriebenen Bestimmungsgleichungen berechnet werden.

\subsection{Weitere Superpositionsmöglichkeiten von Grundlösungen}

Ebenso ist es mit diesem Verfahren möglich, einen Halbraum mit zylindrischem Tunnel und einem kugelförmigen Einschnitt an der Halbraumoberfläche zu modellieren. Dafür müssen die drei Fundamentalsysteme Halbraum, Vollraum mit zylinderförmigen Hohlraum und Vollraum mit sphärischem Hohlraum überlagert werden [2]. In jedem Fundamentalsystem bringt man dazu Einheitsspannungszustände an den jeweiligen realen Oberflächen $\Lambda, \Gamma_{C}$ und $\Gamma_{S}$ auf und berechnet die Spannungen an den jeweils zugehörigen fiktiven Oberflächen $\delta \Lambda, \delta \Gamma_{c}$ und $\delta \Gamma_{S}$. Mit Hilfe der Randbedingungen an der Halbraum-, Zylinder- und Kugeloberfläche erhält man eine geschlossene Lösung der Form:

$$
\begin{aligned}
& C_{i z}(o, s) \hat{\sigma}_{i z}(o, s)+\sum_{o} \sum_{N} \sum_{J} C_{j r}(o, n) \hat{\sigma}_{i z}^{(j r, o n)}(o, s) \sum_{M} \sum_{L} \sum_{K} C_{r k}(m, l) \hat{\sigma}_{i z}^{(r k, m l)}(o, s)=-\hat{p}_{i, \Lambda}(o, s) \\
& \sum_{o} \sum_{S} \sum_{I} C_{i z}(o, s) \hat{\sigma}_{j r}^{(i z, o s)}(o, n)+C_{j r}(o, n) \hat{\sigma}_{j r}(o, n)+\sum_{M} \sum_{L} \sum_{K} C_{r k}(m, l) \hat{\sigma}_{j r}^{(r k, m l)}(o, n)=-\hat{p}_{j, \Gamma_{C}}(o, n) \\
& \sum_{O} \sum_{S} \sum_{I} C_{i z}(o, s) \hat{\sigma}_{r k}^{(i z, o s)}(m, l)+\sum_{o} \sum_{N} \sum_{J} C_{j r}(o, n) \hat{\sigma}_{r k}^{(j r, o n)}(m, l)+C_{r k}(m, l) \hat{\sigma}_{r k}(m, l)=-\hat{p}_{k, \Gamma_{S}}(m, l)
\end{aligned}
$$

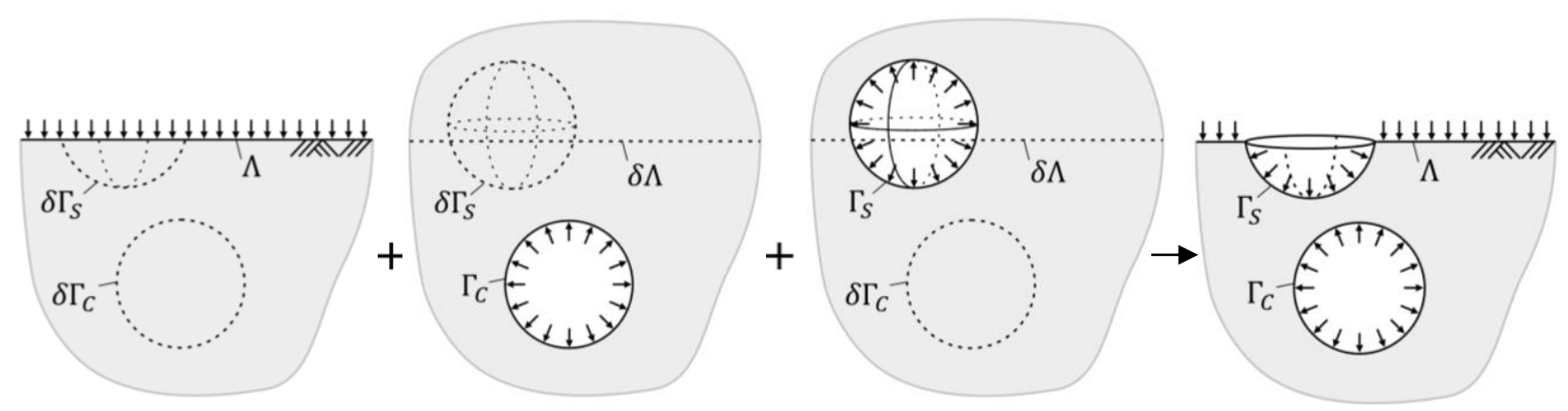

Abbildung 5: Superposition von Fundamentalsystemen zum Halbraum mit Tunnel und sphärischem Einschnitt. 
Mit diesem Lösungsansatz können analog weitere Systeme aus Superpositionen von Fundamentalsystemen hergeleitet werden, wie z.B. ein Halbraum mit zwei parallelen Tunneln oder ein zylindrischer bzw. sphärischer Hohlraum unterhalb einer Bodenschichtung [5].

\subsection{Validierung}

Zur Validierung der zuvor beschriebenen Kopplungsmethode wird ein Halbraum mit einem sehr flachen kugelförmigen Einschnitt $\left(f_{E}=0,1 \mathrm{~m}, R_{0}=6,0 \mathrm{~m}\right)$ mit einer harmonischen Last $p_{R R}\left(d_{l}=1,8 m, f=30 \mathrm{~Hz}\right)$ beaufschlagt, welche entlang der Oberfläche des Einschnitts radial wirkt. Die Ergebnisse dieser Berechnung werden mit der Lösung des Fundamentalsystems Halbraum unter einer äquivalenten, rechteckigen, harmonischen Last $p_{z z}$ verglichen.

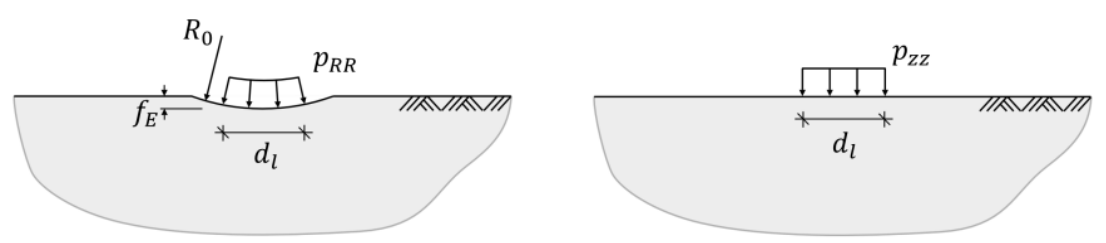

Abbildung 6: Halbraum mit flacher Kugelförmiger Einsenkung (links) und Halbraum (rechts).

Die elastischen Bodenparameter wurden zu $E=206 \cdot 10^{6}\left[\mathrm{~N} / \mathrm{m}^{2}\right]$ und $v=0,3$ gewählt, sowie eine Dämpfung mit einem Verlustfaktor von $\zeta=0,10$ angesetzt. In Abbildung 7 und 8 sind die vertikalen Verschiebungen $u_{z}$ an der Oberfläche für beide Systeme dargestellt. Die Verschiebungen am Punkt $x=0, y=0$ können mit dem gekoppelten System nicht direkt berechnet werden, da die Verschiebungen nur auf den diskretisierten Breitengraden der Kugel ausgewertet werden können. Eine direkte Berechnung des Werts am Pol der Kugel ist nicht möglich.
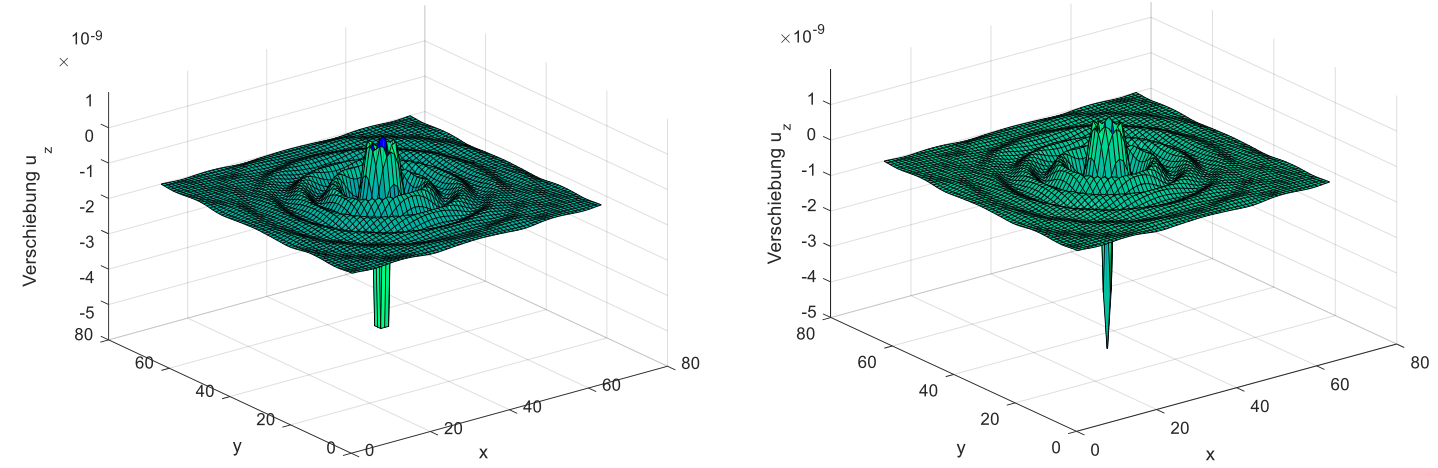

Abbildung 7: $u_{z}$ an der Oberfläche für Halbraum mit flacher kugelförmiger Einsenkung (links) und Halbraum (rechts).

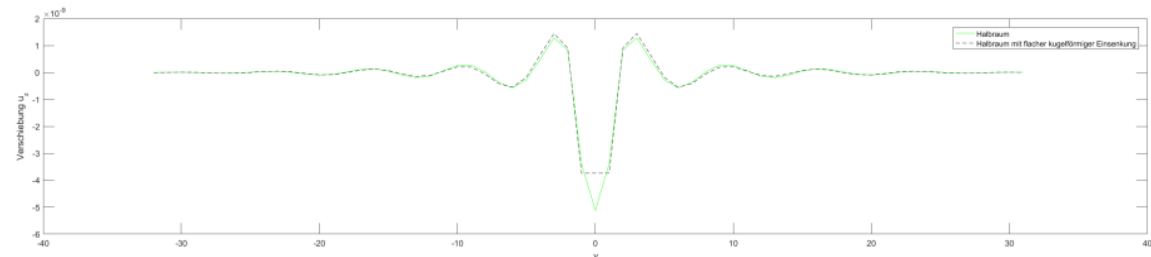

Abbildung 8: $u_{z}$ bei $(\mathrm{z}=0, \mathrm{x}=0)$ für Halbraum mit flacher kugelförmiger Einsenkung (links) und Halbraum (rechts). 
Abbildung 8 zeigt außerhalb des Pols eine sehr gute Übereinstimmung der Ergebnisse für die Lösung des Fundamentalsystems Halbraum und des gekoppelten Systems.

\section{Kopplung mit der Finiten Elemente Methode}

In Abschnitt 3.1 wurde gezeigt wie sich die Steifigkeitsmatrix für einen Halbraum mit sphärischem Hohlraum oder Einschnitt bestimmen lässt.
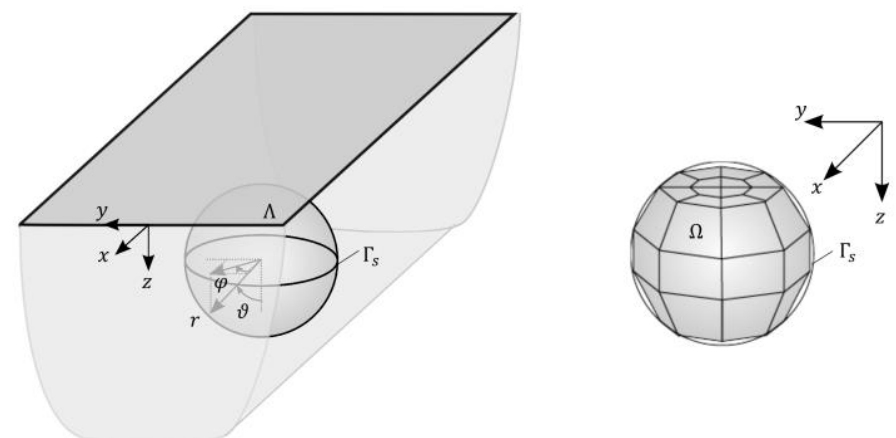

Abbildung 9: Kopplung von Halbraum mit sphärischem Hohlraum (ITM) (links) und FEM Modell (rechts).

Nun können beliebige Strukturen mit der FEM modelliert werden und anschließend, unter Berücksichtigung der Kompatibilität der Verschiebungen und des Kräftegleichgewichts an der kugelförmigen Kopplungsoberfläche, mit der ITM gekoppelt werden [6]. Zur Durchführung der Kopplung müssen die Parameter von beiden Substrukturen in der gleichen Basis beschrieben sein. Daher werden die FEM Freiheitsgrade an der sphärischen Kopplungsoberfläche zunächst in Kugelkoordinaten transformiert und danach in eine Reihe von Kugelflächenfunktionen zerlegt. Für die Kopplung mit dem ITM System müssen dann nur noch die entsprechenden Steifigkeitsterme der beiden Subsysteme addiert werden. Das Gesamtsystem kann durch die dynamischen Steifigkeiten wie folgt beschrieben werden:

$$
\left[\begin{array}{ccc}
K_{\Lambda \Lambda, I T M} & K_{\Lambda \Gamma, I T M} & 0 \\
K_{\Lambda \Gamma, I T M} & K_{\Gamma \Gamma, I T M}+K_{\Gamma \Gamma, \mathrm{FEM}} & K_{\Gamma \Omega, \mathrm{FEM}} \\
0 & K_{\Gamma \Omega, \mathrm{FEM}} & K_{\Omega \Omega, \mathrm{FEM}}
\end{array}\right]\left(\begin{array}{c}
u_{\Lambda, \mathrm{ITM}} \\
u_{\Gamma} \\
u_{\Omega, \mathrm{FEM}}
\end{array}\right)=\left(\begin{array}{c}
p_{\Lambda, \mathrm{ITM}} \\
p_{\Gamma} \\
p_{\Omega, \mathrm{FEM}}
\end{array}\right)
$$

\section{Numerisches Beispiel einer Erschütterungsreduktionsmaßnahme}

Als Beispiel für eine Erschütterungsreduktionsmaßnahme im Übertragungsweg wurde ein offener Schlitz innerhalb des zylinderförmigen FEM Gebiets simuliert, welches an das durch die ITM beschriebene Bodenmodell gekoppelt wurde. Es werden die Verschiebungen an der Halbraumoberfläche infolge einer harmonischen Blocklast mit und ohne Schlitz verglichen (Amplitudenreduktionsfaktor (ARF): $A_{r}=A_{S c h} / A_{0}$ ) [6]. Die Boden- und Lastparameter wurden zu $E=260 \cdot 10^{5}\left[\frac{\mathrm{N}}{\mathrm{m}^{2}}\right], v=0,3, \rho=2000\left[\frac{\mathrm{kg}}{\mathrm{m}^{3}}\right], \zeta=0,1$ und $f=20 \mathrm{~Hz}$ gewählt. Der ARF $A_{r, a v}$ wurde entlang einer Linie auf der lastabgewandten Seite des Schlitzes bei $x=0$ zwischen 
$y=0$ und $y=32,75 m \approx 10 \cdot \lambda_{R}$ ausgewertet sowie $A_{r, a v, \text { surf }}$ innerhalb eines Halbkreises mit Radius $10 \cdot \lambda_{R}$. Abbildung 10 zeigt die deutliche Zunahme der Reduktionswirkung mit steigender Schlitztiefe $d_{t} / \lambda_{R}$ in Abhängigkeit der Rayleighwellenlänge $\lambda_{R}$.
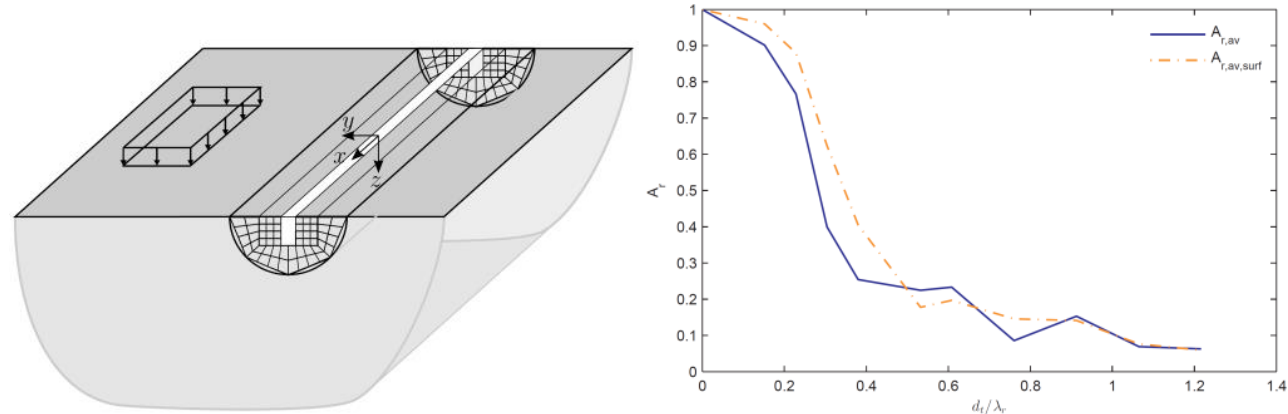

Abbildung 10: Amplitudenreduktionsfaktor $A_{r}$ für $u_{z}$ an Halbraumoberfläche für offenen unendlichen Schlitz in Abhängigkeit der Tiefe des Schlitzes $d_{t}$ normalisiert mit der Rayleighwellenlänge $\lambda_{R}=3,29 \mathrm{~m}$ (vgl.[6]).

\section{Zusammenfassung}

Mit Hilfe der ITM wird eine semi-analytische Lösung für einen Halbraum mit sphärischen oder zylindrischem Hohlraum, Graben oder kugelförmigen Einschnitt im Wellenzahl-Frequenz Bereich hergeleitet, mit welcher wellenzahlabhängige Nachgiebigkeiten an den jeweiligen Oberflächen bestimmt werden können. Diese Nachgiebigkeiten können dann mit der FEM gekoppelt werden und so sehr komplexe Systeme modelliert werden. Damit ist es auch möglich, die dynamische Boden Bauwerk Interaktion abzubilden und Erschütterungsprognosen zu treffen.

\section{Literatur}

[1] E. Kausel, Fundamental Solutions in Elastodynamics. Cambridge University Press, New York, 2006.

[2] G. Frühe, Überlagerung von Grundlösungen in der Elastodynamik zur Behandlung der dynamischen Tunnel-Boden-Bauwerk-Interaktion, Shaker Verlag, 2010.

[3] A.C. Eringen, E.S. Suhubi, Elastodynamics, Vol I: Finite Motions, Vol II: Linear Theory. Academic Press, New York (1975).

[4] G. Müller, Ein Verfahren zur Erfassung der Fundament-Boden-Wechselwirkungen unter Einwirkung periodischer Lasten. Disseration, TU München, Germany, 1989.

[5] J. Freisinger, Modellierung eines geschichteten Halbraums mit sphärischem Hohlraum. Master Thesis, TU München, 2016.

[6] M. Hackenberg, A Coupled Integral Transform Method - Finite Element Method Approach to Model the Soil-Structure-Interaction. Dissertation, TU München, 2016. 